Escuela de Ciencias Sociales y Humanidades, UNED, C.R. URL: http://investiga.uned.ac.cr/revistas/index.php/espiga/index ISSN: 1409-4002 - e-ISSN: 2215-454X

\title{
Construcción de imaginarios sociales en torno a la trata de personas en Costa Rica: estado de la cuestión e ideas para su abordaje
}

\author{
Esteban Gerardo Alfaro Orozco* (D) http://orcid.org/0000-0002-5587-1343 \\ Guillermo Acuña González** (D) http://orcid.org/0000-0002-9196-0300 \\ Cynthia Mora Izaguirre*** (iD) http://orcid.org/0000-0001-8182-1502 \\ Nery Chaves García**** (D) http://orcid.org/0000-0002-3288-1549 \\ Laura Solís Bastos***** (D) http://orcid.org/0000-0002-7434-221X
}

Recibido: 14 de junio, 2017 - Aceptado: 27 de setiembre, 2017

Formato de citación según APA

Alfaro-Orozco E. G., Acuña-González G., Mora-Izaguirre C., Chaves-García N. y SolísBastos L. (2017). Construcción de imaginarios sociales en torno a la trata de personas en Costa Rica: estado de la cuestión e ideas para su abordaje. Revista Espiga, 16(34), 231-254. Doi: http://dx.doi.org/10.22458/re.v17i34.1815

\section{Formato de citación según Chicago-Deusto}

Alfaro-Orozco Esteban Gerardo, Guillermo Acuña-González, Cynthia Mora-Izaguirre, Nery Chaves-García y Laura Solís-Bastos. «Construcción de imaginarios sociales en torno a la trata de personas en Costa Rica: estado de la cuestión e ideas para su abordaje». Revista Espiga, 16, n. ${ }^{\circ} 34$ (julio-diciembre 2017): 231-254. Doi: http:// dx.doi.org/10.22458/re.v17i34.1815

* Licenciado en Psicología. Universidad Nacional. Costa Rica. Correo: estebanalfaroorozco@gmail.com

** Sociólogo, máster en Comunicación Social, Universidad de Costa Rica. Académico del Instituto de Estudios Sociales en Población (IDESPO). Universidad Nacional. Costa Rica.

Correo: guillermo.acuna.gonzalez@una.cr

*** Comunicadora, doctora en Ciencias Políticas de la Universidad de Rostock y máster en dicha área de la Universidad de Costa Rica. Académica del Instituto de Estudios Sociales en Población (IDESPO). Universidad Nacional. Costa Rica. Correo: cynthiamora2000@yahoo.com

**** Licenciada en Relaciones Internacionales con énfasis en Política Exterior y Diplomacia. Universidad Nacional. Costa Rica. Correo: nchavesg@gmail.com

***** Socióloga, máster en Estudios Latinoamericanos con énfasis en Cultura y Desarrollo por el Instituto de Estudios Latinoamericanos de la Universidad Nacional. Investigadora del Instituto de Estudios Sociales en Población (IDESPO). Universidad Nacional. Costa Rica. Correo: laura.solis.bastos@una.cr 


\section{RESUMEN}

La trata de personas es un proceso reconocido de forma contemporánea como uno de los fenómenos sociales de mayor complejidad a nivel global, tanto por el desconocimiento de la población al respecto, como por la dificultad conceptual para su reconocimiento, gracias a la clandestinidad en la cual se desarrolla. Esto ha permitido que sea confundida con otros fenómenos sociales como el tráfico de personas u otros procesos migratorios globales. A su vez, los contextos sociales en los que emerge la trata de personas han permitido identificar cómo cualquier persona a nivel global podría llegar a ser víctima de trata, y como también existen condiciones estructurales que podrían favorecer que algunas personas con características particulares tengan mayor vulnerabilidad social ante este fenómeno.

Palabras clave: trata de personas, derechos humanos, percepciones, vulnerabilidad.

\section{Introducción}

La trata de personas, conceptualmente, ha sido considerada como un delito denominado como la esclavitud del siglo XXI. Sin embargo también ha sido catalogado como uno de los negocios más lucrativos a nivel global, pero a costa de la dignidad humana, poniendo precio y convirtiendo a las personas en objetos del mercado.

La comprensión de este fenómeno social, que no es nuevo ni reciente, no ha sido simple y ha pasado por un largo trayecto para que actualmente sea reconocido a nivel conceptual en todas sus dimensiones, a partir de la firma de una serie de documentos normativos internacionales de forma previa:

- Acuerdo internacional sobre represión de la trata de blancas (1904)

- Convención internacional para la represión de la trata de blancas (1910)

- Convenio internacional para la represión de trata de mujeres mayores de edad (1933)

- Convención para la supresión del tráfico de personas y de la explotación de prostitución ajena (1949) 1

La firma de estos documentos normativos internacionales fue un antecedente importante en la conceptualización de la trata de personas a nivel internacional y además el reconocer la amplitud que debía contener en el marco de las dimensiones que lo involucran. En el artículo 3. ${ }^{\circ}$ del Protocolo de Palermo 2000, en el Protocolo Adicional de la Convención de las Naciones Unidas contra la Delincuencia Organizada Transnacional relativos a la Prevención, Represión y Castigo de la trata de personas, especialmente de Mujeres y Niños, ha sido definida así:

Por «trata de personas» se entenderá la captación, el transporte, el traslado, la acogida o la recepción de personas, recurriendo a la amenaza o el uso de la fuerza u otras formas de coacción, al rapto, al fraude, al engaño, al abuso de poder o de una situación de vulnerabilidad o a la concesión o recepción de pagos o beneficios para obtener el consentimiento de una persona que tenga autoridad sobre otra con fines de explotación. Esa explotación incluirá, como mínimo, la explotación de la prostitución ajena u otras formas de explotación sexual, los

1. Gissele Gutiérrez, «Curso introductorio sobre protección de poblaciones migrantes en condición de vulnerabilidad», Manual para el facilitador (OIM: OIM, 2012), 9-76. 
trabajos o servicios forzados, la esclavitud o prácticas análogas a la esclavitud, la servidumbre o la extracción de órganos. Etenizia Maria, Gonçalves Araújo. Trata de personas, un enfoque analítico de la legislación penal brasileña ${ }^{2}$.

Como menciona la definición, la trata de personas es un delito de mucha complejidad y multidimensional en el que se induce paulatinamente a la persona a convertirse en una víctima y su humanidad se mercantiliza. Este artículo aborda la construcción de percepciones y valoraciones de la población costarricense sobre el concepto, las causas, el flujo de información y otra serie de variables existentes alrededor del fenómeno de la trata de personas en el país.

\section{Caracterización de Costa Rica en torno a la trata de personas}

Para la comprensión del fenómeno de la trata de personas, se debe reconocer que si bien cualquier persona a nivel global podría llegar a ser víctima de trata, hay razones particulares que permiten determinar distintas causas de vulnerabilidad en las poblaciones y que podrían eventualmente ser causa de condiciones de riesgo.

\section{Condiciones geográficas}

Desde el punto de vista geográfico, Costa Rica es un país ubicado en Centroamérica, de tan sólo $51100 \mathrm{~km}^{2}$ de área total de territorio nacional; el país se subdivide en siete provincias (San José, Alajuela, Cartago, Heredia, Guanacaste, Puntarenas y Limón), concentra la mayor parte de su población en la región central de país, clasificada como la zona urbana. A nivel fronterizo territorial comparte límites geográficos con Panamá y Nicaragua; y a nivel marítimo con Panamá, Colombia y Ecuador.

Desde el punto de vista migratorio, se ha caracterizado por mantener flujos de recepción de migrantes, primordialmente nicaragüenses, y en segundo lugar colombianos; sin embargo, a su vez se presenta como un país de migración de paso hacia Estados Unidos; de forma reciente en el 2015 y 2016 fue notoria la presencia de migrantes extra regionales, lo cual marca la pauta de variaciones en las dinámicas migratorias del país.

Sin embargo, es reconocido el papel tan relevante que tienen las fronteras al interior del fenómeno, en el tanto la trata de personas puede ocurrir tanto al interior del país como fuera de él; sin embargo una de las principales formas de captación se muestra a través de diversas ofertas en el extranjero, tal como se señala en un informe del Departamento de Estado de Estados Unidos, Informe sobre trata de personas (2005), esto citado por la Organización Internacional para las Migraciones (OIM) ${ }^{3}$.

2. María José Magliano y Janneth Clavijo. «La trata de personas como una forma abusiva de migración: securitización del debate migratorio y criminalización del sujeto migrante». Revista Aportes andinos. Revista electrónica de derechos humanos (2014): 9. En http://repositorio.uasb.edu.ec/bitstream/10644/3341/1/ RAA-31\%20Mar $\%$ C3\%ADa $\% 20 \mathrm{Jos} \% \mathrm{C3} \%$ A9\%20Magliano $\% 20 \mathrm{y} \% 20 \mathrm{Janneth} \% 20$ Clavijo.pdf

3. Organización Internacional para las Migraciones (OIM). Trata de personas. Aspectos básicos. (Organización Internacional para las Migraciones, 2006). Edición en PDF, 19-25. 


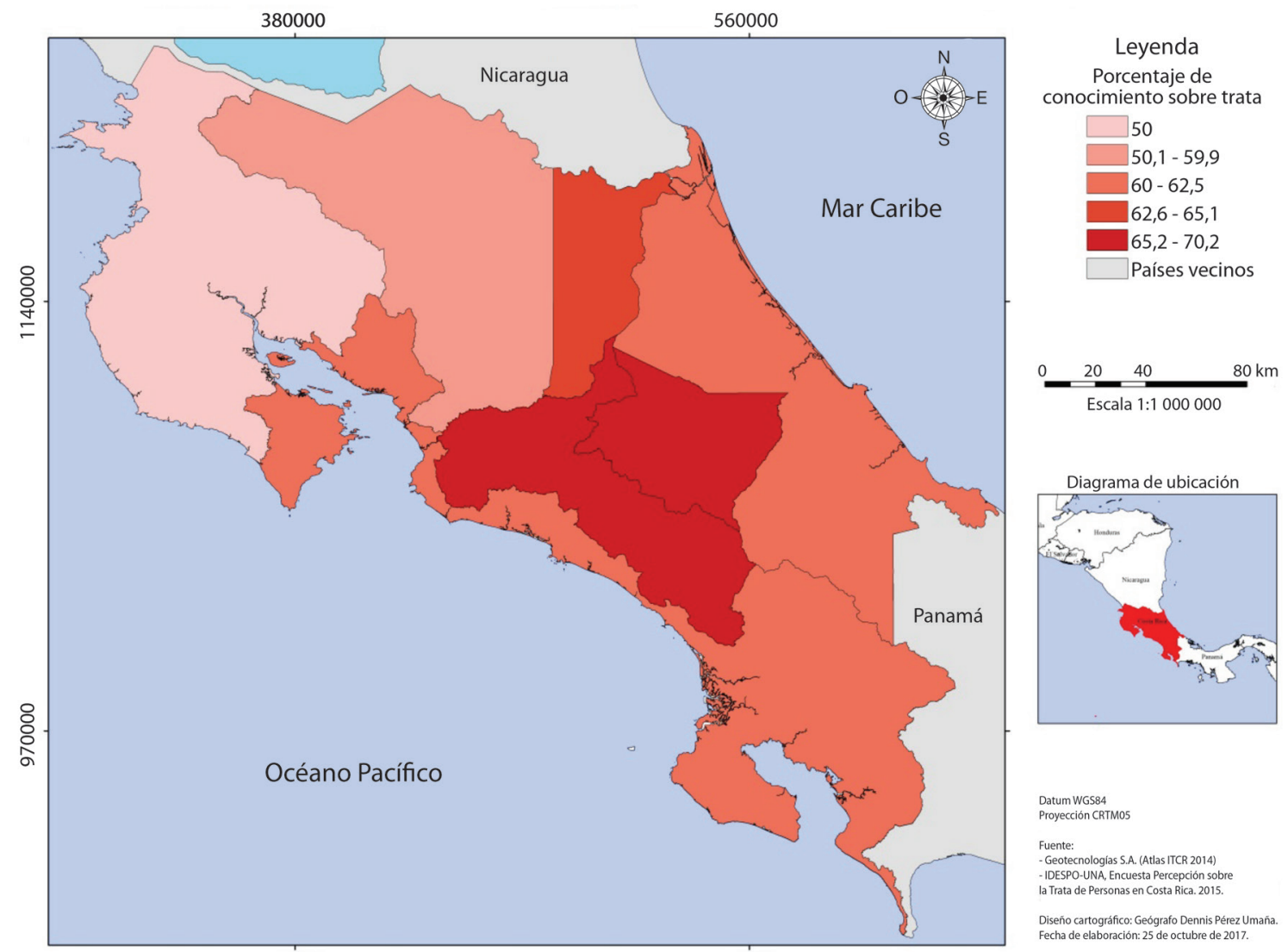

Figura 1. Valores porcentuales acerca del conocimiento de la trata de personas por provincia, 2015. Fuente: IDESPO-UNA, encuesta Percepción sobre la trata de personas en Costa Rica, 2015. Distribución geográfica por provincias de la población encuestada acerca del conocimiento de la trata de personas en Costa Rica, agosto, 2015.

De acuerdo con el Informe Anual sobre Trata de Personas del Departamento de Estado de Estados Unidos, cada año entre 600000 y 800000 personas cruzan las fronteras internacionales como víctimas de trata; de esta cifra, el ochenta por ciento son mujeres y niñas y el cincuenta por ciento son personas menores de edad ${ }^{4}$.

La mejor herramienta de prevención ante este delito que denigra la humanidad de quienes lo han padecido, es la información y el reconocimiento de estar frente a un peligro evidente. En el siguiente mapa se muestra una asociación entre el conocimiento sobre el tema en la población costarricense encuestada por IDESPO en 2015 y la ubicación espacial en el territorio nacional.

4. Organización Internacional para las Migraciones (OIM), Trata de personas..., 19-25. 


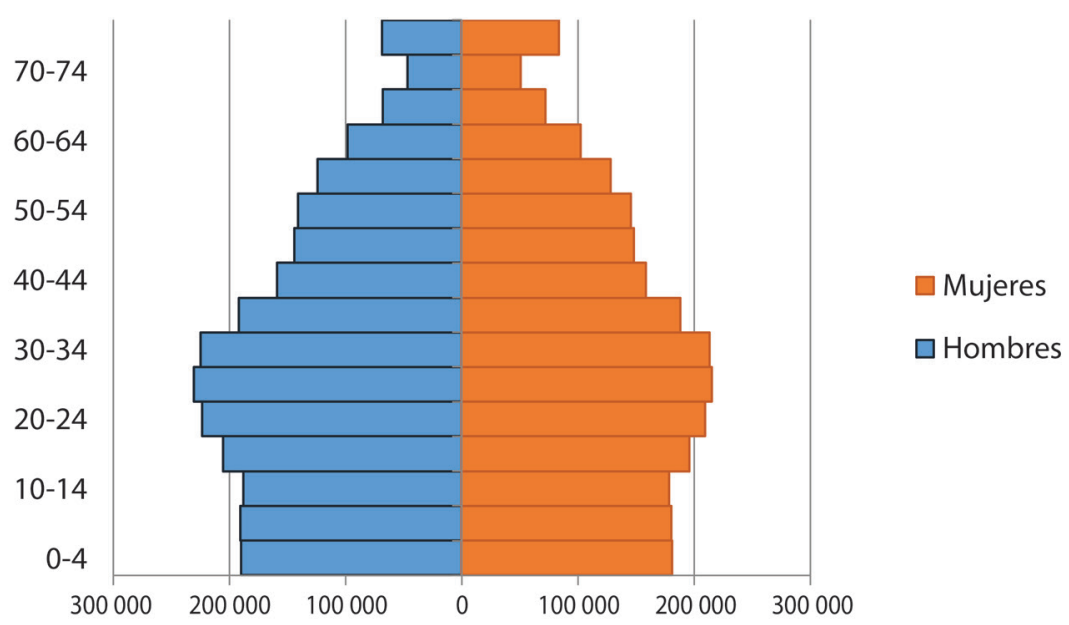

Figura 2. Estructura de la población nacional de Costa Rica al año 2017. Fuente: Procesado por L. Solís Bastos (2017), basado en proyecciones de población INEC-Costa Rica a través de Redatam+SP de la CEPAL/CELADE.

En el mapa se observa que el menor conocimiento acerca de la trata de personas coincide con los espacios transfronterizos del país, por lo cual esta podría ser una condición de vulnerabilidad para la población de estos territorios, aunada a menores índices de desarrollo humano, bajas condiciones socioeconómicas y educativas, así como menores ofertas de empleo y condiciones productivas, esto en comparación con la región central del país.

\section{Condiciones demográficas}

Se ha estimado que la población total de Costa Rica asciende a 4947490 habitantes, según proyección realizada a junio de 2017 por el INEC Costa Rica ${ }^{5}$.

Al observar estos datos es de suma relevancia identificar que del total de población, 742239 son niños/as, 768320 son personas adolescentes, 1705495 son mujeres en edad productiva, mientras que en el caso de los hombres asciende a 1743670 y finalmente 389833 son personas adultas mayores; sin embargo, para el fenómeno en particular se ha reconocido que las principales víctimas tienden a ser las personas menores de edad y las mujeres en edad productiva y reproductiva.

En cuanto a aspectos sociodemográficos, el país, para el 2016, se encuentra marcado por un evidente proceso de transición demográfica avanzada, según datos de $\mathrm{INEC}^{6}$, publicados en la página web oficial, es posible observar características como las siguientes: en el caso de la natalidad por una Tasa Bruta de Natalidad con tendencia a la baja, de

5. Instituto de Estadística y Censos (INEC). Estimaciones y proyecciones de población por sexo y grupos de edad. 1950-2050. (INEC, 2017), en http://www.inec.go.cr/

6. Instituto de Estadística y Censos (INEC). Estadísticas Vitales 2016. (INEC, 2017).

En http://www.inec.go.cr/ 
14,31 nacimientos por cada mil habitantes; mientras que en el caso de la esperanza de vida, esta se muestra en aumento, siendo de 80 años; por otra parte para la mortalidad, la Tasa Bruta de Mortalidad fue de 4,62 personas por cada 1000 habitantes, asociada al incremento de población adulta mayor presente en la estructura poblacional; finalmente en el caso de la tasa de mortalidad infantil esta también se encuentra con tendencia a la baja, con una tasa de 7,93 defunciones de niños/as menores de un año por cada 1000 nacidos vivos.

Condiciones como las anteriores, desde el punto de vista demográfico, muestran un desarrollo poblacional favorable para el país, similar al de países catalogados con un nivel avanzado de desarrollo; por lo cual la presencia de población emigrante en edad productiva podría ser considerada como favorable desde el punto de vista del llamado bono demográfico.

\section{Condición económica}

En cuanto a las características socioeconómicas, este país muestra que la desigualdad de la distribución de los ingresos de las personas en la sociedad costarricense en los últimos cinco años, se ha mantenido relativamente estable, con un coeficiente de Gini actual por persona de 0,52 , mientras que el porcentaje de hogares pobres asciende únicamente al 6,30\%, en el cual su ingreso per cápita está por debajo de la línea de pobreza extrema; mientras que para el caso de pobreza, corresponde a $20,50 \%$, cuyo ingreso per cápita está por debajo de la línea de pobreza extrema ${ }^{7}$.

En referencia a otras características socioeconómicas, a continuación se muestran las tendencias observadas en vinculación a empleo publicadas por el INEC ${ }^{8}$. Para el 2016 la tasa de ocupación evidenciaba que 54 personas por cada 100 en edad de trabajar se encontraba laborando; en tanto la tasa de desempleo abierto era de 9,7 por cada 100 personas mayores de 15 años; por otra parte se observa una evidente brecha de género en el desempleo en donde el desempleo es casi un $48 \%$ más alto en las mujeres que los hombres.

El panorama económico actual ha generado que el país pueda percibirse como una zona de atracción para la población emigrante, razón por la cual las condiciones laborales, económicas y sociales se vislumbran favorables ante escenarios de oportunidad.

\section{Metodología}

El principal insumo empleado para el análisis de la construcción de los imaginarios de la población costarricense sobre la trata de personas fue la encuesta realizada de forma telefónica a nivel nacional, durante agosto de 2015, con el objetivo de conocer las percepciones de la población en Costa Rica sobre la trata de personas.

7. Instituto de Estadística y Censos (INEC). Encuesta Nacional de Hogares 2016. (INEC, 2017).

En http://www.inec.go.cr/

8. Instituto de Estadística y Censos (INEC). Encuesta Continua de Empleo 2016. (INEC, 2017). En http://www.inec.go.cr/ 
El cuestionario elaborado para la ejecución de este instrumento se compuso de 22 preguntas agrupadas en diferentes módulos enfocados en temas como:

- Conocimiento sobre trata de personas.

- Acceso a la información sobre trata de personas.

- Fenómenos sociales asociados a la trata de personas en Costa Rica.

- Características sociodemográficas de la población encuestada.

La encuesta telefónica se realizó a 800 personas, la selección de teléfonos se hizo al azar y además se llevó a cabo por cuotas probabilísticas de sexo y edad. Cuenta con un nivel de confianza del 95\% y un margen de error de 3.5\%.

En cuanto a las características de la población encuestada, se seleccionaron personas costarricenses o extranjeras con dos o más años de residir en el país, mayores de dieciocho años y habitantes de viviendas particulares que poseen teléfono residencial.

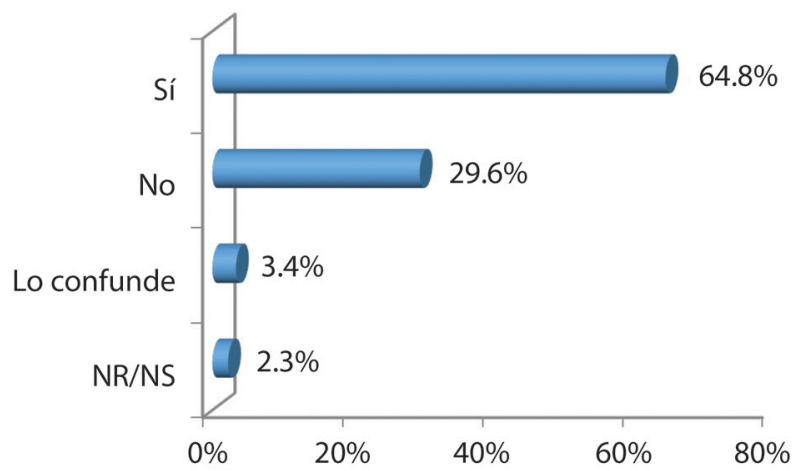

Figura 3. Distribución porcentual del conocimiento de la población encuestada sobre el concepto «trata de personas» (n=800), agosto 2015. Fuente: IDESPO-UNA, encuesta percepción sobre la trata de personas en Costa Rica, 2015. Distribución porcentual del conocimiento de la población encuestada sobre el concepto «trata de personas» (n=800), agosto 2015.

Para el análisis de los datos obtenidos mediante la encuesta, se realizó una evaluación descriptiva de las principales variables, por medio de distribuciones de frecuencias relativas de las percepciones de la población encuestada sobre la trata de personas en Costa Rica.

\section{Percepciones sobre el concepto trata de personas}

Como se indicó, la definición de trata es aceptada a nivel internacional, pero para una parte de la población global este concepto se mantiene ausente, o confuso, en el tanto se desconoce esta acepción; es así como resulta relevante reconocer cómo se construye el imaginario de la población costarricense, específicamente sobre la trata de personas en el país.

De acuerdo con el gráfico 2, casi un $65 \%$ de la población encuestada afirma conocer qué es trata de personas; sin embargo, se debe destacar que persiste un bajo porcentaje que confunde el término con otros fenómenos sociales, generalmente con el tráfico ilícito de migrantes y la prostitución voluntaria. 
TABLA 1

\begin{tabular}{ll}
\cline { 2 - 2 } Acciones & Refiere a la captación, el transporte/traslado y la acogida o recepción de personas. \\
\cline { 2 - 2 } Fines & $\begin{array}{l}\text { Incluye amenaza, uso de la fuerza u otras formas de coacción, rapto, fraude, engaño, abuso de poder } \\
\text { o de una situación de vulnerabilidad y concesión o recepción de pagos o beneficios para obtener el } \\
\text { consentimiento de una persona que tenga autoridad sobre otra. }\end{array}$ \\
\cline { 2 - 2 } & $\begin{array}{l}\text { Prostitución ajena u otras formas de explotación sexual, trabajos o servicios forzados, esclavitud, } \\
\text { prácticas análogas a la esclavitud, servidumbre y extracción de órganos. }\end{array}$ \\
\hline
\end{tabular}

Fuente: Gissele Gutiérrez, curso introductorio sobre protección de poblaciones migrantes en condición de vulnerabilidad, Manual para el facilitador, Tomo 2, OIM, 2012, 9-76. Componentes de la trata de personas.

Por sus características particulares en la trata de personas suele ser difícil identificar que el hecho que ha ocurrido puede calificarse como tal, pero a su vez también complejiza la identificación de las personas víctimas y de los victimarios de este tipo de delito.

Por lo que para comprender claramente el concepto de trata, es necesario identificarlo como un proceso que tiene diferentes etapas y componentes, como se muestra en la siguiente tabla:

A la población encuestada que indicó conocer qué es trata de personas, se le aplicó la siguiente pregunta: ¿Me podría indicar qué entiende por trata de personas?, se obtuvieron los siguientes resultados que se muestran en la tabla 2.

TABLA 2

\begin{tabular}{lc}
\multicolumn{1}{c}{ Definición conceptual } & Porcentaje \\
\hline Explotación de personas & 58,2 \\
Explotación sexual & 15,4 \\
Traslado de personas a otros lugares & 20,3 \\
No se comprende del todo el término & 3,8 \\
NS/NR & 0,2 \\
Otros & 2,0 \\
\hline Total & 100 \\
\hline
\end{tabular}

Fuente: IDESPO-UNA, encuesta Percepción sobre la trata de personas en Costa Rica, 2015. Distribución porcentual de la población encuestada sobre la construcción del concepto «trata de personas» $(n=800)$, agosto, 2015.

Como se identificó, el concepto es muy amplio, así como las dimensiones para su conocimiento; se debe tener la claridad de que en el marco de las percepciones para quienes logran identificar el fenómeno, suele haber una tendencia común de asociarlo con situaciones muy específicas; sin embargo, la clave para comprender la claridad del concepto mencionado por cada persona encuestada, se analizó a partir de la referencia a la mención de la siguiente idea general: la condición de obligar a una persona a hacer algo en contra de su voluntad o consentimiento, con fines de explotación de diversos tipos, por medio de mecanismos como engaños, amenazas y hasta la privación de la libertad. 
En ese sentido, resulta fundamental clarificar conceptualmente, con base en lo estipulado por Naciones Unidas, así como otras organizaciones sociopolíticas acerca del fenómeno trabajado en este artículo. Como se puede ver en la tabla 2, hay algunas confusiones sobre los delitos de: trata de personas, de tráfico ilícito de personas, así como el trabajo sexual. En la tabla 3 se detallan las distintas características de los fenómenos.

TABLA 3

\begin{tabular}{|ll}
\hline \multicolumn{1}{c}{ Concepto } & \multicolumn{1}{c}{ Definición } \\
Trata de personas & $\begin{array}{l}\text { Se refiere al sometimiento de una persona para realizar distintos } \\
\text { trabajos forzados (fines) en contra de su voluntad. }\end{array}$ \\
Tráfico ilícito de personas & $\begin{array}{l}\text { Traslado de personas de un estado a otro, o más, de formas ilícitas. } \\
\text { Trabajo sexual }\end{array}$ \\
$\begin{array}{l}\text { Refiere a la libre elección de personas a ejercer el trabajo sexual } \\
\text { como una opción laboral y/o subsistencia. Al ser una decisión } \\
\text { personal y no un sometimiento por otros (as) y/o organizaciones } \\
\text { criminales; el trabajo sexual no es trata de personas. }\end{array}$
\end{tabular}

Fuente: elaboración propia con base en el Alto Comisionado de Naciones Unidas para Refugiados (ACNUR) y La Sala Organización de Trabajadoras y ex Trabajadoras Sexuales-Costa Rica. Definición conceptual de trata de personas, tráfico ilícito de personas y trabajo sexual. Noviembre, 2017.

De acuerdo con la tabla 3, el sometimiento de otras personas resulta la clave determinante para diferenciar el trabajo sexual de la trata de personas. Además, el tráfico ilícito de personas refiere específicamente al traslado de personas de un estado a otro por medios que no se encuentran en la legislación correspondiente.

Para el estudio de las percepciones sobre la trata de personas y la forma en la que se construye el imaginario social, fue necesario formular la siguiente pregunta: ¿Me podría dar tres ejemplos de formas en las que se da la trata de personas? Esto para identificar las principales asociaciones con los tipos de trata, ya que si bien son múltiples las manifestaciones, e incluso una misma persona puede llegar a ser explotada de distintas formas, era relevante poder dimensionar cuáles eran las más mencionadas, en el tanto estas son las que persisten tanto en la construcción simbólica del concepto, así como en su reconocimiento como caso particular.

TABLA 4

\begin{tabular}{lc}
\multicolumn{1}{c}{ Ejemplos de trata de personas } & Porcentaje \\
Trata con fines de explotación sexual & 41,2 \\
Trata con fines de trabajo forzoso & 31,6 \\
Trata con fines de tráfico de drogas & 7,2 \\
Trata con fines de extracción y venta de órganos & 19,9 \\
Total & 100 \\
\hline
\end{tabular}

Fuente: IDESPO-UNA, encuesta Percepción sobre la trata de personas en Costa Rica, 2015. Distribución porcentual de la población encuestada sobre la percepción de tipos de trata de personas que ocurren en Costa Rica (n=454), agosto, 2015. 
De acuerdo con las principales respuestas obtenidas, la principal forma de trata que reconoce la población encuestada es la trata con fines de explotación sexual, seguida por la asociada al trabajo forzoso; si bien estas son las más mencionadas, cabe destacar que en menor porcentaje se presentan otras asociadas al tráfico de drogas y a la extracción y venta de órganos, que como se menciona «esto aplica para comprender la evolución que este delito ha tenido en términos de su vinculación con los intereses, mecanismos y estrategias con otras modalidades de crimen organizado como el narcotráfico, el tráfico ilícito de migrantes, el terrorismo, entre otros ${ }^{9}$.

\section{Percepciones sobre la vulnerabilidad a la trata de personas}

En el marco de la consulta, también se indagó el conocimiento respecto a los factores que vulnerabilizan a la población para ser sometidos como víctimas de la trata, así como su percepción respecto del perfil de las víctimas según edad, sexo, movilidad geográfica y acceso al empleo o la educación.

Para analizar estas percepciones, resulta necesario entender el trasfondo socioeconómico de la trata de personas como un fenómeno social que se ha agravado en la última década en Latinoamérica, en la medida en que se han diversificado las características de los medios, víctimas y victimarios de esta actividad ilícita ${ }^{10}$. Este trasfondo es analizado por Abraham Sánchez Ruiz ${ }^{11}$, quien ofrece una lectura de la trata de personas como un fenómeno social que esencialmente surge como consecuencia de la mercantilización del ser humano como mercancía, motivada por el afán de enriquecimiento económico a través de relaciones productivas donde media el uso de la violencia directa o indirecta y la dominación como métodos para explotar el excedente de producción de las víctimas por parte de los tratantes; y donde la actividad se realiza de manera ilícita frente a las legislaciones estatales e internacionales en derechos humanos, mas no escapando de los determinantes de un mercado capitalista, neoliberal y globalizado.

Esta perspectiva permite acercarse a visualizar el fenómeno como una actividad humana de enriquecimiento ilícito, hecha posible en el desarrollo de la economía neoliberal donde se establecen relaciones entre vendedores y consumidores específicos; con esto se genera una demanda por servicios o bienes humanos igual de específicos y no adquiribles a la luz del juicio público, sostenido a través de un proceso de compraventa de mercancías humanas (visto en las tres fases de la trata de personas: enganche, traslado y control, y consumación de la explotación), en el cual una doble moral estatal e interestatal ejercida en la falta de acciones políticas y económicas suficientes para fortalecer la defensa y vigilancia activa de los derechos humanos de las personas, se ve

9. Gissele, Gutiérrez. «Curso introductorio sobre protección de poblaciones migrantes en condición de vulnerabilidad», Manual para el facilitador (OIM: OIM, 2012), 25-26.

10. Oficina de las Naciones Unidas contra la Droga y el Delito (UNODC). UNODC Global Report on Trafficking in Persons (Naciones Unidas: Oficina de Drogas y Crimen de las Naciones Unidas, 2016), edición en PDF, 89-97.

11. Abraham, Sánchez Ruiz. «Nueve tesis esenciales sobre la trata de personas y el falso compromiso de la democracia con los derechos humanos». XVI Certamen de ensayo sobre derechos humanos. Trata de personas (2017): 11-52. En: http://www.codhem.org.mx/LocalUser/codhem.org/difus/16certamenlibro.pdf 
implicada en el fortalecimiento de las ventajas competitivas y comparativas de esta forma de enriquecimiento ${ }^{12}$.

Por lo tanto, teniendo claro el trasfondo, hay un acercamiento a las situaciones contextuales y/o coyunturales que pueden incidir en el desarrollo de la trata según la percepción de las personas encuestadas. Entonces se planteó: «Quisiéramos saber su opinión sobre: ¿Cuánto influyen las siguientes situaciones para que en el país se den casos de trata de personas? $\gg^{13}$. Los resultados respectivos a esta pregunta se visualizan en la tabla 5.

TABLA 5

\begin{tabular}{lcccc} 
Factores que influyen para que se den en el país casos de trata de personas & Sí & No & NS/NR & Total \\
\hline Limitaciones para estudiar (económicas, distancia geográfica, transporte) & 79,2 & 20,4 & 0,4 & $100 \%$ \\
Escasas oportunidades de empleo & 89,9 & 9,7 & 0,4 & $100 \%$ \\
Empleo informal (empleo sin contrato ni garantías sociales) & 76,5 & 22,3 & 1,2 & $100 \%$ \\
Migraciones internacionales & 91,5 & 6,8 & 1,7 & $100 \%$ \\
Pobreza & 94,2 & 5,6 & 0,2 & $100 \%$ \\
Turismo & 68,5 & 28 & 3,5 & $100 \%$ \\
Profesionales sin oportunidades de empleo en lo que estudió & 53,2 & 45 & 1,7 & $100 \%$ \\
Crimen organizado & 92,8 & 4,7 & 2,5 & $100 \%$ \\
\hline
\end{tabular}

Fuente: IDESPO-UNA, encuesta Percepción sobre la trata de personas en Costa Rica, 2015. Distribución porcentual de la población encuestada sobre la percepción de los factores que incluyen para que se den casos de la trata de personas en Costa Rica (n=515), agosto, 2015.

De acuerdo con la percepción de las personas encuestadas, las principales causas de la trata son pobreza $(94,2 \%)$, crimen organizado $(92,8 \%)$, migraciones internacionales $(91,5 \%)$ y escasas oportunidades de empleo $(89,9 \%)$. Por el contrario, las limitaciones para estudiar $(79,2 \%)$, el empleo informal $(76,5 \%)$, el turismo $(68,5 \%)$ y las escasas oportunidades de trabajo con que cuentan los profesionales en su campo de estudio $(53,2 \%)$, responden a los menores porcentaje de importancia, siendo este último a la vez, la opción con mayor porcentaje de respuestas negativas al respecto de la pregunta realizada.

A partir de estas respuestas, exceptuando el reconocimiento del crimen organizado y las migraciones internacionales en Costa Rica (factores que abordaremos a posteriori), se enumeran una serie de condiciones socioeconómicas vulnerables como principales factores para que se den casos de trata según la percepción de las personas encuestadas (pobreza, escasas oportunidades de empleo e incluso las limitaciones para acceder al estudio). Las respuestas apuntan al análisis de la trata como un fenómeno social que se desenvuelve principalmente por la vulnerabilización socioeconómica de la

\footnotetext{
12. Ibíd., 11-52.

13. Instituto de Estudios Sociales en Población (IDESPO). Presentación de análisis de resultados de la encuesta: «Percepción sobre la trata de personas en Costa Rica». (Costa Rica: Universidad Nacional de Costa Rica, 2015), 7.
} 
sociedad, donde según Sánchez ${ }^{14}$ apunta a dos aspectos claves para analizar la trata en los países latinoamericanos.

El primero de estos aspectos refiere a que la vulnerabilización masiva de las mayorías en países latinoamericanos, más específicamente de las víctimas que pasan a ser parte de las mercancías humanas explotadas por las personas tratantes, representa parte de las ventajas competitivas de su negocio en la región. El mejor reflejo de este aspecto en el caso costarricense, al identificar que las poblaciones con mayor cantidad de casos de víctimas de este fenómeno, son las mujeres y jóvenes ${ }^{15}$; a su vez, son quienes figuran como las poblaciones en peores condiciones de solvencia económica, con menor acceso al desarrollo de emprendimientos propios, su acceso a empleos se concentra en el sector de baja calificación y/o informal (donde incluso los niños pueden verse vulnerados por el contexto y dinámica socioeconómica de sus familias) o registrados como en inactividad económica por causa del desempleo ${ }^{16}$.

Segundo, que la trata de personas si bien es analizada como un fenómeno que violenta los derechos humanos de primera generación (equidad de género, derechos de la niñez y adolescencia, entre otros), es principalmente impulsada por la violación y desatención del cumplimiento de derechos humanos de segunda y tercera generación por parte de los Estados latinoamericanos (derecho a vida digna, derecho a la educación, derechos laborales, derechos de los pueblos, entre otros); esto a través del empobrecimiento socioeconómico de la población que ensancha los flujos migratorios motivados por razones laborales, limitando el acceso de las familias con menores ingresos a oportunidades educativas, lo cual merma sus recursos culturales para identificar o reclamar sus plenos derechos.

Una forma de ejemplificar la forma mediante la cual la vulnerabilización de los derechos económicos, sociales y culturales en las poblaciones con menos recursos socioeconómicos profundiza el fenómeno de la trata, se produce en la situación de la región Chorotega, la cual es identificada como la zona de mayor flujo de casos y redes de trata de personas a través de los años ${ }^{17,18}$ y 19 y a la vez como una de las regiones con mayores y sostenidos índices de desigualdad y pobreza ${ }^{20}$.

14. Abraham, Sánchez Ruiz. «Nueve tesis...», 11-52.

15. U.S. Department of State (Ed.). Trafficking in Persons Report 2016 (Estados Unidos de América: Departamento de Estado de los Estados Unidos de América, 2016). Edición en PDF, 140-142.

16. Programa Estado de la Nación en Desarrollo Humano Sostenible (Ed.). Vigesimosegundo Informe Estado de la Nación en Desarrollo Humano Sostenible (Costa Rica: Programa Estado de la Nación en Desarrollo Humano Sostenible, 2016), Edición en PDF, 85-119.

17. U.S. Department of State (Ed.). Trafficking in Persons ..., 140-142.

18. U.S. Department of State (Ed.). Trafficking in Persons ..., 131-132.

19. U.S. Department of State (Ed.). Trafficking in Persons ..., 144-145.

20. Programa Estado de la Nación en Desarrollo Humano Sostenible (Ed.). Vigesimosegundo Informe..., 85-119. 
Esta lectura además es reforzada por las respuestas de las personas encuestadas en el momento en que se preguntó: «¿Cuál es la razón que puede volver más vulnerable a una persona para convertirse en víctima de trata de personas? ${ }^{21} \gg$

A partir de las respuestas brindadas por las personas encuestadas, se construyeron categorías de agrupación, generando resultados que reflejan esta forma de ponderar la vulnerabilización socioeconómica de la población como principal coadyuvante para la victimización de personas a ser afectadas por tratantes. Los resultados son visibles en la tabla 6.

TABLA 6

\begin{tabular}{lc} 
Causas de vulnerabilidad ante la trata de personas & Porcentaje \\
Pobreza & 45,5 \\
Desempleo & 12,3 \\
Ignorancia/falta de información & 10,6 \\
Falta de educación & 9,6 \\
Porque son niños/jóvenes & 1,2 \\
Desintegración familiar & 3,7 \\
Por migrar & 1,7 \\
Exceso de confianza & 4,2 \\
Inseguridad & 1,9 \\
Ambición & 4,5 \\
Otros & 4 \\
NS/NR & 0,8 \\
Total & 100 \\
\hline
\end{tabular}

Fuente: IDESPO-UNA, encuesta Percepción sobre la trata de personas en Costa Rica, 2015. Distribución porcentual de la población encuestada sobre la percepción acerca de la principal causa de vulnerabilidad de las personas que podrían ser víctimas de trata de personas en Costa Rica ( $\mathrm{n}=515)$, agosto, 2015.

La pobreza repunta en la percepción de la población encuestada como la principal causa de vulnerabilidad ante la trata de personas con un $45,5 \%$, seguido del desempleo el cual constituye la segunda causa percibida con un 12,3\%, le siguen además la ignorancia y/o falta de información (10,6\%). En contraposición la desintegración familiar (3,7\%), la inseguridad $(1,9 \%)$ y por migrar $(1,7 \%)$ responden a los porcentajes menores.

21. Instituto de Estudios Sociales en Población (IDESPO). Presentación de análisis de resultados de la encuesta: «Percepción sobre la trata de personas en Costa Rica». (Costa Rica: Universidad Nacional de Costa Rica, 2015), 7. 
Como otros factores identificados con significancia porcentual por la población encuestada, refieren el crimen organizado y las migraciones internacionales; sin embargo, para el análisis de estos aspectos como factores de la trata de personas, cabe contextualizarlos en la caracterización del perfil de las víctimas de este fenómeno social.

Por lo tanto, en las percepciones de las personas encuestadas, la distribución de la población en función de su condición etaria, de género y condición socio-económica inciden como factores en el nivel de riesgo de las personas a ser víctimas. Las personas encuestadas señalaron que las víctimas potenciales serían las personas adolescentes $(80,8 \%)$, niños y niñas $(80,4 \%)$ y mujeres $(78,6)$, así como las personas migrantes quienes tienen un $73,0 \%$ de mucha vulnerabilidad, las personas desempleadas cuentan con un $54,2 \%$ y los hombres $(18,6 \%)$, quienes no reflejan una mayor vulnerabilidad en las percepciones recogidas.

TABLA 7

\begin{tabular}{lcccccc}
\multicolumn{1}{c}{ Nivel de riesgo según población } & Mucho & Algo & Poco & Nada & NS/NR & Total \\
Niños y niñas & 80,4 & 11,7 & 7,0 & 0,6 & 0,4 & $100 \%$ \\
Adolescentes & 80,8 & 14 & 4,9 & 0,2 & 0,2 & $100 \%$ \\
Personas jóvenes (18-35 años) & 46,4 & 34,2 & 17,3 & 1,9 & 0,2 & $100 \%$ \\
Mujeres & 78,6 & 13,6 & 7 & 0,4 & 0,4 & $100 \%$ \\
Hombres & 18,6 & 38,6 & 38,6 & 3,1 & 1 & $100 \%$ \\
Personas migrantes & 73 & 15,5 & 9,9 & 0,6 & 1 & $100 \%$ \\
Personas desempleadas & 54,2 & 27,4 & 15,9 & 2,1 & 0,4 & $100 \%$ \\
Personas con bajo nivel educativo & 67,6 & 19,8 & 10,1 & 2,1 & 0,4 & $100 \%$
\end{tabular}

Fuente: IDESPO-UNA, encuesta Percepción sobre la trata de personas en Costa Rica, 2015. Distribución porcentual de la población encuestada sobre el nivel de riesgo que tienen las poblaciones de vivir una situación de trata de personas en Costa Rica (n=515), agosto 2015 .

Las mujeres, adolescentes, niños y niñas y las personas migrantes en el país son percibidas como principales sujetos de riesgo a ser víctimas de tratantes; la vulnerabilidad de estas poblaciones específicas se agrava en un contexto que les expone a condiciones socioeconómicas deficitarias; sin embargo, la situación de riesgo específica de cada grupo deviene de características diferenciadas dentro de la dinámica de enriquecimiento ilícito de este fenómeno social, lo cual se analizará posteriormente.

Lamentablemente, una de las principales dinámicas que fortalecen el riesgo de mujeres y personas menores de edad (PME) a ser víctimas de trata, refiere a las altas rentas, normalización e institucionalización de la explotación sexual y laboral en el país. Las mujeres, niñas y niños se identifican como principales víctimas con fines de explotación sexual y laboral, esto a través de la acentuación de dinámicas como el turismo sexual infantil, el trabajo forzado de mujeres y niños en el sector agro productivo y la coerción psicológica y física ejercida a mujeres trabajadoras del comercio sexual por parte de tratantes, con el fin de sostenerles en una dinámica de explotación sexual ${ }^{22}$.

22. U.S. Department of State (Ed.). Trafficking in Persons ..., 140-142. 
En el caso de la población migrante, es necesario considerar la relación económica intrínseca entre migración laboral y trata de personas. Costa Rica es principalmente un país receptor de personas migrantes de origen centroamericano, cuya razón de movilidad está determinada principalmente por la migración forzada por razones económicas (Programa Estado de la Nación, 2016); sin embargo, la movilidad transfronteriza en condiciones de vulnerabilidad socioeconómica condiciona a las personas a moverse de forma irregular frente a las legislaciones nacionales e internacionales y, por tanto, desprovista de los medios para hacer valer sus derechos.

Por lo tanto, a partir de esta dinámica la población migrante en el país no está solo sujeta a condiciones deficitarias en relación con el acceso a salud, empleo y educación, sino que es además víctima de redes de trata con fines de explotación sexual y laboral en actividades como la agro producción, la construcción, la pesca, el sector de servicios comerciales, el servicio doméstico, el turismo sexual y el turismo sexual infantil ${ }^{23}$.

\section{Percepciones sobre el acceso a información referida a la trata de personas}

El estudio acerca de la construcción de las percepciones pasa en términos generales por el registro de un conjunto de filtros y mediaciones en las cuales los medios de comunicación ejercen un rol fundamental.

La compresión de fenómenos como las migraciones, los movimientos poblacionales, el tráfico de personas y la trata de personas se produce desde distintos ángulos y valoraciones. En el caso de la trata de personas, objeto principal del presente artículo, a menudo existe una confusión sobre su significado, su naturaleza transnacional, sus condicionantes multicausales y sus impactos sobre la dignidad y los derechos humanos de las personas que se exponen a dicha situación.

El error en la concepción podría estar siendo producido, como se verá más adelante, por un déficit en la información con la que cuenta la población en general. Magliano y Clavijo ${ }^{24}$ plantean que las formas de concebir a la trata de personas en la esfera política migratoria regional no se alejan de los postulados de control y seguridad que subyacen a la construcción de esa agenda, reconfigurando y legitimando una concepción de las migraciones y del migrante como problema.

A la larga, los medios de comunicación podrían estar formando parte de esta consideración, ya sea por los contenidos elaborados o porque simplemente la información que comparten no es la adecuada en la formulación de una idea correcta sobre el tema.

Dicho lo anterior, ante la concepción de la trata de personas como problema y amenaza, y entendiéndola solamente desde postulados vinculados con la seguridad sin

23. Ibíd, 140-142.

24. María José Magliano; Janneth Clavijo, «La trata de personas como una forma abusiva de migración: securitización del debate migratorio y criminalización del sujeto migrante». Revista Aportes andinos. Revista electrónica de derechos humanos (2012): 5. En http://repositorio.uasb.edu.ec/bitstream/10644/3341/1/ RAA31 $\% 20$ Mar $\%$ C3\%ADa $\% 20 J$ os $\%$ C3\%A $\%$ 20Magliano $\% 20 y \% 20$ Janneth $\% 20$ Clavijo.pdf 
considerar elementos relacionados con los derechos humanos y la dignidad de las personas, subyacen las respuestas coercitivas, quedando subsumidas las víctimas de la trata de personas como si fueran ellas mismas las productoras del flagelo que experimentan.

Vinculado a esta idea en la encuesta realizada por IDESPO $^{25}$, se consultó sobre los niveles de información que tenía la población costarricense en aquel momento acerca del fenómeno de la trata de personas. De acuerdo con la información generada, existe un nivel bastante bajo de información respecto a la trata de personas en el país, si se suman los valores entre «algo informado, poco informado y nada informado», con un $81,3 \%$ de resultado de las respuestas dadas por las personas se ubicó en dichos rangos.

Esta mención no debería pasar desapercibida en un contexto en que el flujo de información es una constante y se produce a partir de varios soportes y formatos informativos.

La producción noticiosa, por ejemplo, refleja cada tanto notas respecto a casos de trata de personas en Costa Rica y en el resto de la región centroamericana, así como la consolidación de una reciente industria de la producción audiovisual que empieza a preocuparse cada vez más por esta problemática ${ }^{26}$. Sin embargo, se debe indicar la existencia de déficit en la producción de información referente al tema.

En un análisis sobre los enfoques de los medios de comunicación costarricenses, referidos a la migración y el refugio, se señalaba como tendencia temática en los encuadres televisivos al abordaje de la trata de personas, pero con referencias equivocadas y estereotipadas del fenómeno. Apuntaba el informe que «no en pocas ocasiones, a lo largo del año, las noticias televisivas encuadran la trata de personas en abordajes que hacen referencia a otras dinámicas, lo que indica una permanente confusión conceptual sobre este tema, que no solo es resorte de los telenoticieros del país» ${ }^{27}$.

Por otra parte, el conocimiento y la información a la que acceden las personas respecto a la trata de personas son dos aspectos distintos de una misma dimensión, la producción comunicativa frente al fenómeno. Como se ha indicado ya en otro momento en este artículo, la población encuestada alegó conocer sobre la temática, pero paradójicamente se siente poco informada, como quedó evidenciado en las líneas anteriores.

El manejo de la información pareciera entonces no ser el adecuado. La confusión sobre términos como el de trata y tráfico se produce en el marco de la elaboración de pocos contenidos mediáticos y un análisis difuso de los impactos que produce en las personas, las comunidades y las colectividades que lo experimentan.

25. Instituto de Estudios Sociales en Población (IDESPO). Presentación de análisis..., 9.

26. Una muestra de recientes producciones relacionadas al fenómeno de la trata de personas refieren la producción argentina La mosca en la ceniza, del año 2010, dirigida por Gabriela David, sobre la vida de dos amigas del interior argentino que son llevadas mediante engaños al centro del país, a trabajar en actividades sexuales comerciales; el drama Evelyn (2012), dirigida por Isabel de Ocampo y que relata el viaje de una joven peruana a España y cómo cae en redes de explotación sexual comercial en aquel país. Más cercana al contexto regional, la producción mexicana La vida precoz y breve de Sabina Rivas (2012), dirigida por Luis Mandoki, narra el drama de una joven hondureña en su paso por la frontera entre México y Guatemala.

27. CONAMAJ. Prensa costarricense y población migrante y refugiada: los encuadres noticiosos en la prensa escrita y los noticieros de televisión sobre la migración y las personas en condición de refugio en el país (Costa Rica: Informe de noticias del año 2009-CONAMAJ, 2010). Edición en PDF. 
Por otra parte, en la actualidad el acceso a medios de comunicación dibuja una relación más compleja y activa de las audiencias con sus contenidos. Ya no se trata de los medios de comunicación tradicionalmente conocidos hace unos años. Su transformación rápida e intensa ha sido evidente.

De estructuras mediáticas más o menos construidas, han pasado a convertirse en grupos comunicativos por donde pasan los contenidos en distintos formatos. Es decir, por ejemplo, un medio de comunicación impreso (periódico) ha incorporado en sus soportes otras narrativas comunicativas, con la suma de la producción de noticias en tiempo real para que sean consumidas en la inmediatez de las redes sociales. Sobresale así la producción gráfica, audiovisual y radial de una noticia cuya fuente primaria es un diario escrito.

Tomando en cuenta esta dimensión de cambio en los medios como sistemas de información, deben leerse los resultados generados respecto de si se ha escuchado mencionar el tema y en cuáles medios lo han hecho.

En el primer caso, casi un $82 \%$ de la población encuestada indicó haber escuchado sobre la trata de personas, mientras que prácticamente un $18 \%$ respondió negativamente. Este aspecto diferencia tanto los niveles de información como la retención que de ciertos temas realizan las personas a nivel general, tal y como quedó evidenciado en el análisis realizado anteriormente en este mismo apartado.

Por otra parte, existe una exposición mayoritaria a la televisión como el principal medio de comunicación por parte de las personas consultadas, en la cual escucharon alguna referencia al tema o la misma palabra trata de personas $(96,9 \%)$, seguido por internet $(57,3 \%)$ y las redes sociales $(54,5 \%)$. En contraste, la radio no figura entre los medios por los cuales la población encuestada se informa acerca de la trata de personas en el país.

Es posible advertir dos aspectos sobre esta información generada. El primero es el rol de la televisión en la producción de las agendas informativas que se generan en relación con temas como el de la trata de personas. De acuerdo con Humanes (2001), el acontecer noticioso en televisión debe ser analizado desde dos formatos: los episódicos (noticias centradas en eventos específicos o casos particulares) y los temáticos (con una cobertura basada en la explicación y el conocimiento); el primero se basa en acontecimientos concretos que ilustran un tema, mientras que el segundo presenta hechos generales ${ }^{28}$.

De acuerdo con lo señalado, es el esquema episódico el que domina en las noticias en televisión, debido a las constricciones del tiempo, los intereses comerciales, la publicidad y la objetividad. Esto se da sin profundidad, entonces es fácil advertir que las informaciones emanadas desde el constructo televisivo respecto de temas como la trata de personas, presenten una gran debilidad conceptual y explicativa y que sea trasladada a las audiencias que la consumen mayoritariamente, como quedó expuesto en los datos analizados.

28. CONAMAJ. Prensa costarricense y..., 2-5. 
En segundo término, en el peso que presentan las redes sociales como fuentes de información consultadas por la población ante dichos temas, se debe poner especial cuidado sobre los contenidos desarrollados y la forma en que son utilizados por las poblaciones.

\section{Respuesta del estado costarricense frente a la trata y el tráfico}

Como se mencionó al inicio del documento, el Protocolo de Palermo constituyó la herramienta de atención para este delito, ratificado por Costa Rica en el 2002. En el 2005 se formó la Coalición Nacional contra el Tráfico Ilícito de Migrantes y la Trata de Personas (CONATT), instancia en la cual varios representantes de diferentes instituciones $^{29}$ llevaban a cabo coordinaciones y acciones para visibilizar y luchar contra esos dos flagelos.

En el 2013 se logró concretar la Ley Contra la Trata de Personas y creación de la Coalición Nacional contra el Tráfico Ilícito de Migrantes y la Trata de Personas (CONATT), Ley 9095, que vino a ser la base y el apoyo legal de la CONATT, así como de otros lineamientos y acciones que derivan de la ley: «La ley viene a generar la articulación y los recursos para trabajar el tema, antes de la ley no existían los recursos. Se ha tardado algunos años para poner a trabajar y funcionar esos recursos, no ha sido sencilla la tarea que ha tenido el país, cuando hablamos de fondos; es materia complicada la ejecución de fondos en Costa Rica, es muy compleja» ${ }^{30}$.

Como se puede apreciar, es claro que el abordaje institucional del delito específico de trata y tráfico es de relativa corta data; es decir, aproximadamente diez años, lo cual podría influir en que las poblaciones aun no tienen total claridad de qué es la trata y en qué se diferencia del tráfico de personas.

En el marco de dicha ley, y junto a otros actores sociales, desde el 2016 empezó una campaña denominada «Corazón Azul» que cuenta con el apoyo del sector público y privado y consiste en: «Una iniciativa mundial de sensibilización que a través del arte, el deporte y los diálogos con los principales actores de la sociedad, pretende alertar a la comunidad, especialmente jóvenes y niños, acerca del impacto negativo de la trata de personas e incentivar la participación del gobierno e instituciones para sensibilizar a la

29. Instituciones miembros de la CONATT: 1. Caja Costarricense del Seguro Social (CCSS); 2. Consejo Nacional de Rehabilitación y Educación Especial; 3. Dirección General de Migración y Extranjería (DGME); 4. Dirección General de Tránsito; 5. Dirección de Inteligencia y Seguridad Nacional (DIS); 6. Fiscalía General de la República; 7. Instituto de Alcoholismo y Farmacodependencia (IAFA); 8. Instituto Costarricense de Turismo (ICT); 9. Instituto Mixto de Ayuda Social (IMAS); 10. Instituto Nacional de Aprendizaje (INA); 11. Instituto Nacional de la Mujeres (INAMU), 12. Ministerio de Educación (MEP); 13. Ministerio de Gobernación, Policía y Seguridad Pública; 14. Ministerio de Justicia y Paz; 15.Ministerio de Relaciones Exteriores y Culto (RREE); 16. Ministerio de Salud (MS); 17. Ministerio de Trabajo y Seguridad Social (MTSS); 18. Oficina de Atención y Protección a la Víctima del Delito; 19. Organismo de Investigación Judicial (OIJ), 20. Patronato Nacional de la Infancia (PANI); 21. Secretaría Técnica de la Comisión Nacional contra la Explotación Sexual Comercial.

30. Cynthia, Mora Izaguirre. Entrevista con la gestora de la Unidad de Trata y Tráfico, DGME (Rohrmoser, 5 junio 2017). Costa Rica: San José, 2017. 
población costarricense, ya que se dirige a todas las clases sociales, a personas de todas las edades y a todos los sectores $»^{31}$.

Una de las estrategias de dicha campaña es promover el uso externo de un corazón azul como señal de solidaridad:
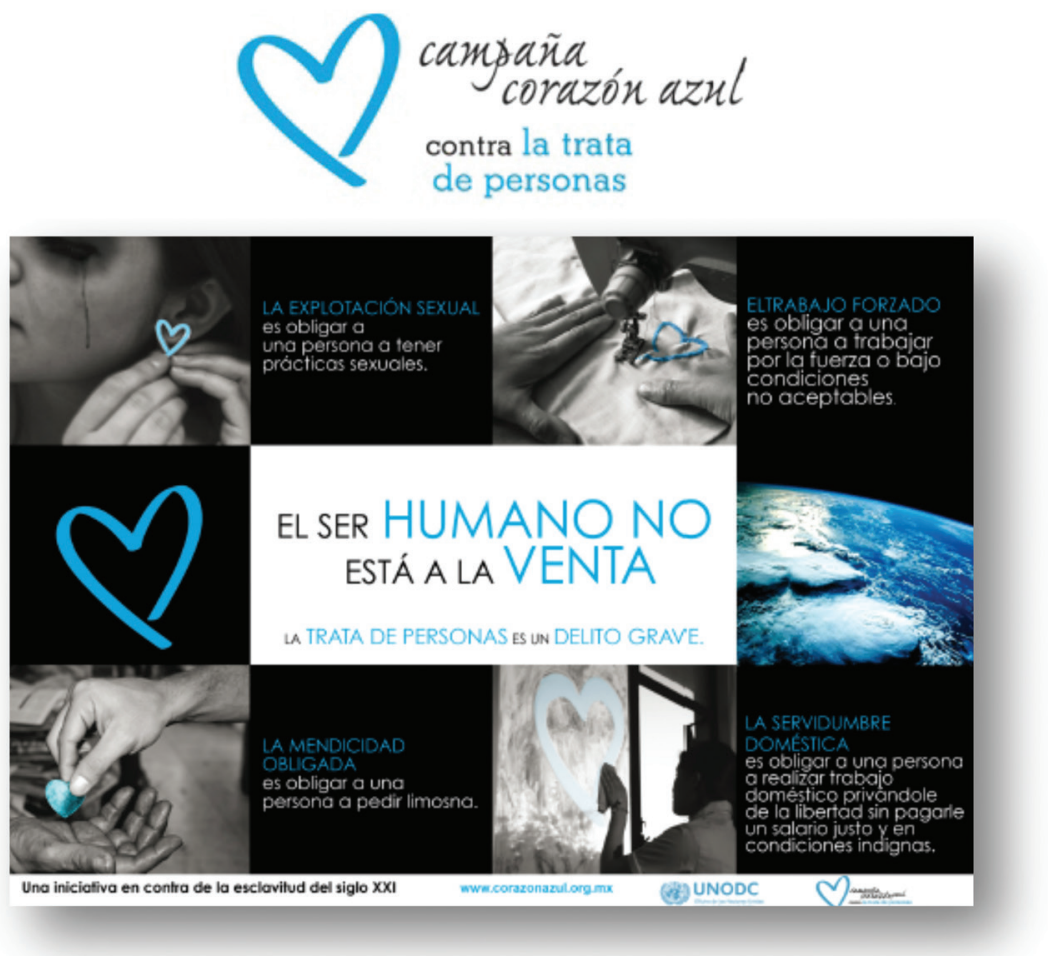

Figura 4. Fuente: UNODC, resumen ejecutivo de la campaña Corazón Azul, 2016.

Yo creo que con Corazón Azul lo vamos a lograr, ese es el objetivo, que la gente conozca, que denuncie, pero también se necesita que el Ministerio Público se ponga pilas y logre sentencias y que demuestre que la trata pasa y es un delito ${ }^{32}$.

Como se puede apreciar, dicha campaña busca posicionar el tema de la trata y el tráfico, pero constituye solo un esfuerzo entre muchos más que deberían darse desde el Estado por medio de sus diferentes instancias.

Cabe señalar que las instituciones sí cuentan con los recursos para dichas tareas y coordinaciones, pues se cuenta con financiamiento por medio del Fondo Nacional contra la Trata de Persona y el Tráfico Ilícito de Migrantes (FONATT), que se alimenta de un

31. Oficina de las Naciones Unidas contra la Droga y el Delito (UNODC). Resumen Ejecutivo de la Campaña Corazón Azul. Costa Rica: San José, 2016.

32. Cynthia, Mora Izaguirre. Entrevista con la gestora... 
dólar en el impuesto de salida del país que se ampara a la Ley 8316, Ley Reguladora de los Derechos de Salida del Territorio Nacional.

Sobre el tema de las víctimas, el reto que tiene que enfrentar el Estado es la penalización y visibilización de este delito, pero «del 2010 al 2017 el país registró a 162 víctimas, según información del Equipo de Repuesta Inmediata (ERI) (...). No obstante, la Oficina de las Naciones Unidas contra la Droga y el Delito (UNODC) calcula que por cada víctima identificada existen 20 más sin identificar, lo que nos daría una cifra cercana a 3 240 casos en Costa Rica desde el 2010» (La Nación, 2 julio 2017).

Pero la realidad dicta que para el sistema judicial ha sido complejo emitir sentencias y, como lo señaló Chaves en el 2015, «ello es fundamental para luchar contra este flagelo» ${ }^{33}$. La Nación además publicó que desde el 2009, que se tipificó la trata como delito,

\section{Muchos afectados, pocas sentencias}

Los que sufren por trata de personas en Costa Rica afrontaron explotación laboral y en su mayoría proceden de países de Centroamérica y Asia.

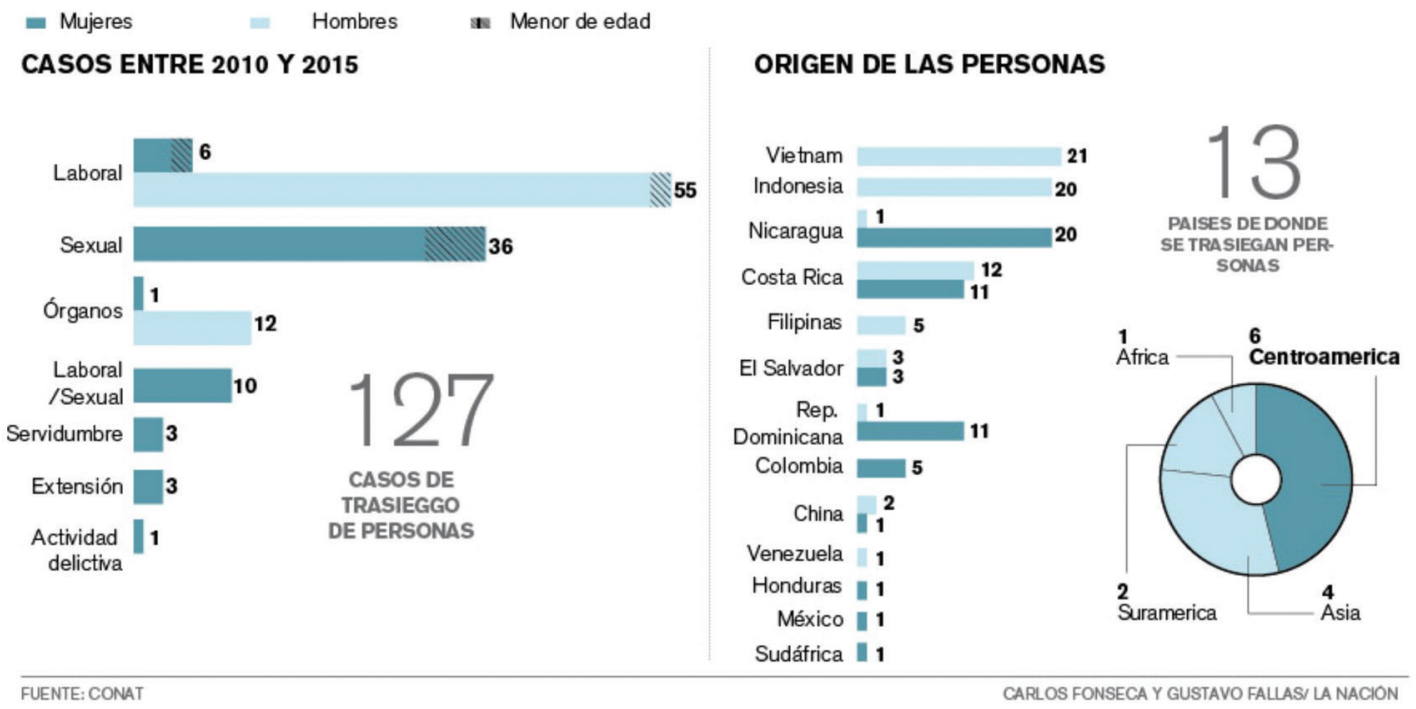

Figura 5. Fuente: Gustavo Fallas. Ley contra la trata de personas solo dio ocho sentencias en seis años. La Nación, lunes 25 setiembre, 2015. Acceso: 15 de noviembre, 2017. En: http://www.nacion.com/sucesos/seguridad/Ley-trata-personas-sentenciasanos_0_1514248593.html

33. Gustavo Fallas. «Ley contra la trata de personas solo dio 8 sentencias en 6 años». La Nación, viernes 25 setiembre, 2015. Acceso: 15 de noviembre, 2017. http://www.nacion.com/sucesos/seguridad/Ley-tratapersonas-sentencias-anos_0_1514248593.html 
solo se produjeron ocho sentencias a partir de las 127 víctimas reconocidas. Casi dos años después de esa publicación, el número de sentencias sigue siendo el mismo pese a que el número de víctimas subió a 162, según información brindada por la CONATT $^{34}$.

A la fecha, el país tiene un reto muy complejo por asumir, pues debe posicionar el tema dentro de la población para lograr denuncias, ya sea de las víctimas o de personas que sospechen que alguien es víctima, pero también pasa por la capacitación de los funcionarios, la atención de víctimas, judicialización de los implicados y respectivas sentencias, en un panorama tan complejo, el consuelo es que el Estado tiene recursos para asumir el reto por medio del FONATT.

\section{Conclusiones}

Uno de los objetivos de este tipo de trabajo es conocer las percepciones que tiene la población nacional, en este caso de la trata de personas, lo cual permite ver que el Estado sí ha logrado posicionar el tema, pero evidenciando la necesidad nacional por trabajar en una mayor delimitación y definición clara del delito para que sea más fácil su detección y de esta manera promover la denuncia.

El Estado tiene una gran oportunidad por contar con el marco legal y apoyo económico, no solo para posicionar el tema por medio de campañas o capacitaciones a funcionarios, sino también de ayudar a las personas víctimas a rehacer sus vidas, lo cual es una oportunidad de desarrollo incluso de núcleos familiares enteros.

Por otra parte, es necesario evidenciar que la desigualdad cada vez más pronunciada que vive Costa Rica y la región, es un medio para facilitar que las personas sean víctimas de trata en su ilusión o necesidad de satisfacer sus necesidades y la de sus familias.

La problemática de una agenda político-económica a nivel estatal que fortalece la flexibilización laboral y la escasez de fuentes de empleo, arroja a la población al sector laboral informal que los expone gradualmente a diferentes formas de explotación o a la migración forzada por razones laborales.

Ese panorama desatiende las necesidades y derechos de los pueblos y mina la independencia económica y política, lo cual les sostiene en una condición de vulnerabilidad socioeconómica y propicia condiciones idóneas para que la persona se convierta en víctima.

Para evidenciar esa problemática, no solo debe trabajar el Estado, también los medios de comunicación. Un desafío en esta dirección indica la necesidad de mejorar los contenidos noticiosos y la construcción de agenda sobre estas temáticas, con información depurada, actualizada y de primera fuente, mediada por personas especialistas que orienten de mejor manera el desarrollo de abordajes teóricos y empíricos más adecuados.

34. José Pablo Román. «Costa Rica: tierra fértil para esclavos». La Nación, domingo 2 de julio, 2017. Acceso: 15 de noviembre, 2017. En: http://www.nacion.com/nacional/derechos-humanos/Costa-Rica-tierra-fertilesclavos_0_1642835780.html 
En un contexto de diversidad de fuentes en redes sociales, es fácil confundir las que producen información científica y empíricamente verificable, de las que son establecidas desde ángulos superficiales, ideológicos y poco sistemáticos.

El Estado y la sociedad en general son los responsables de promover tanto el conocimiento de que la trata es un delito como de inculcar una actitud de denuncia ante este; es por ello que insumos como el de reconocer las percepciones de la población sobre el tema de la trata de personas se pueden transformar en línea base para plantear estrategias de acción y de comunicación en todos los ámbitos abordaje.

\section{ABSTRACT}

\section{Structuring the social archetypal around human trafficking in Costa Rica: status of the issue and ideas for its approach}

Human trafficking is a contemporary known process, one of the social phenomena with greater complexity worldwide, due to the lack of awareness of the population as well as its conceptual difficulty for its acknowledgment thanks to the secrecy in which it develops. This has helped to confuse it with other social phenomena such as global migratory processes. In turn, the social contexts in which human trafficking emerges, have allowed to identify how any person worldwide can become a victim of this phenomenon. Also, the fact that there are structural conditions which would favor that some people with specific characteristics have greater social vulnerability facing this phenomenon has emerged.

Key words: human trafficking, human rights, perceptions, vulnerability.

\section{RÉSUMÉ}

\section{La construction des imaginaires sociaux autour de la traite des êtres humains} au Costa Rica: état des lieux et idées pour l'aborder

La traite des êtres humains est un processus contemporain reconnu comme l'un des phénomènes sociaux d'une extrême complexité au niveau mondial tant pour la méconnaissance de la population sur ce sujet que pour la difficulté conceptuelle pour sa reconnaissance du fait de la clandestinité dans laquelle est développée. En raison de cela, la traite des êtres humains est souvent confondue avec d'autres phénomènes sociaux tels que le trafic des êtres humains ou d'autres processus liés aux migrations globales. À leur tour, les contextes sociaux où émerge la traite des êtres humains ont permis d'identifier comme toute personne dans le monde pourrait devenir une victime de ce processus ; et en même temps, l'existence des conditions structurales qui pourraient favoriser que certaines personnes qui présentent des caractéristiques particulières soient plus vulnérables à ce phénomène.

Mots-clés: traite des êtres humains, droits de l'homme, perception, vulnérabilité.

\section{Bibliografía}

CONAMAJ. Prensa costarricense y población migrante y refugiada: los encuadres noticiosos en la prensa escrita y los noticieros de televisión sobre la migración y las personas en condición de refugio en el país. Costa Rica: Informe de noticias del año 2009-CONAMAJ, 2010. Edición en PDF. 
Fallas, Gustavo. «Ley contra la trata de personas solo dio 8 sentencias en 6 años». La Nación, viernes 25 setiembre, 2015. Acceso: 15 de noviembre, 2017. http://www.nacion.com/sucesos/seguridad/Ley-trata-personassentencias-anos_0_1514248593.html

Gonçalves Araújo, Etenizia Maria. Trata de personas, un enfoque analítico de la legislación penal brasileña. España: Universidad Autónoma de Barcelona, 2012. En https://ddd.uab.cat/pub/trerecpro/2012/ hd1_2072_203161/treball_recerca_egoncalves.pdf

Gutiérrez, Gissele. «Curso introductorio sobre protección de poblaciones migrantes en condición de vulnerabilidad», Manual para el facilitador, Tomo 2. OIM: OIM, 2012. Edición en PDF.

INEC, Costa Rica (2017) INEC, Encuesta Continua de Empleo 2016. Recuperado de http://www.inec.go.cr/

INEC, Costa Rica (2017) INEC, Encuesta Nacional de Hogares 2016. Recuperado de http://www.inec.go.cr/

INEC, Costa Rica (2017) INEC, Estadisticas Vitales 2016. Recuperado de http://www.inec.go.cr/

INEC, Costa Rica (2017) INEC, Estimaciones y proyecciones de población por sexo y grupos de edad. 1950-2050. Recuperado de http://www.inec.go.cr/

Instituto de Estudios Sociales en Población (IDESPO). Presentación de análisis de resultados de la encuesta: «Percepción sobre la Trata de Personas en Costa Rica». Heredia, Costa Rica: Universidad Nacional de Costa Rica, 2015.

Ley Contra la Trata de Personas y creación de la Coalición Nacional contra el Tráfico Ilícito de Migrantes y la Trata de Personas (CONATT), Ley 9095, (2013).

Magliano, María José; Janneth Clavijo. «La trata de personas como una forma abusiva de migración: securitización del debate migratorio y criminalización del sujeto migrante.» Revista Aportes andinos. Revista electrónica de derechos humanos. Ecuador: Universidad Andina Simón Bolivar, n. 31 (2012), http://repositorio.uasb.edu.ec/bitstream/10644/3341/1/RAA-31\%20Mar $\%$ C3\%ADa $\% 20$ Jos $\%$ C3\%A9\%20Magliano $\% 20 y \% 20$ Janneth $\% 20$ Clavijo.pdf

Mora Izaguirre, Cynthia. Entrevista con la gestora Sandra Chaves de la Unidad de Trata y Tráfico, DGME (Rohrmoser, 5 junio 2017). Costa Rica: San José, 2017.

Oficina de las Naciones Unidas contra la Droga y el Delito (UNODC). Resumen Ejecutivo de la Campaña Corazón Azul. Costa Rica: San José, 2016.

Organización Internacional para las Migraciones (2006) Trata de Personas Aspectos Básicos, recuperado de https://www.oas.org/atip/reports/trata.aspectos.basicos.pdf

Programa Estado de la Nación en Desarrollo Humano Sostenible (Ed.). Vigesimosegundo Informe Estado de la Nación en Desarrollo Humano Sostenible. San José, Costa Rica: Programa Estado de la Nación en Desarrollo Humano Sostenible, 2016. En http://estadonacion.or.cr/22/

Román, José Pablo. «Costa Rica: tierra fértil para esclavos». La Nación, domingo 2 de julio, 2017. Acceso: 15 de noviembre, 2017. http://www.nacion.com/nacional/derechos-humanos/Costa-Rica-tierra-fertil-esclavos_0_1642835780.html

Sánchez Ruiz, Abraham. «Nueve tesis esenciales sobre la trata de personas y el falso compromiso de la democracia con los derechos humanos.» XVI Certamen de ensayo sobre derechos humanos. Trata de personas (2017): 11-52, http://www.codhem.org.mx/LocalUser/codhem.org/difus/16certamenlibro.pdf

U.S. Department of State (Ed.). Trafficking in Persons Report 2014. Estados Unidos de América: Departamento de Estado de los Estados Unidos de América, 2014. En https://www.state.gov/j/tip/rls/tiprpt/2014/ index.htm 
U.S. Department of State (Ed.). Trafficking in Persons Report 2015. Estados Unidos de América: Departamento de Estado de los Estados Unidos de América, 2015. En https://www.state.gov/j/tip/rls/tiprpt/2015/ index.htm

U.S. Department of State (Ed.). Trafficking in Persons Report 2016. Estados Unidos de América: Departamento de Estado de los Estados Unidos de América, 2016. En https://www.state.gov/j/tip/rls/tiprpt/2016/ index.htm

United Nations Office on Drugs and Crime (Ed.). UNODC Global Report on Trafficking in Persons. Naciones Unidas: Oficina de Drogas y Crimen de las Naciones Unidas, 2016. En https://www.unodc.org/unode/ data-and-analysis/glotip.html 